\title{
Trastornos de ansiedad según distribución por edad, género, variaciones por regiones, edad de aparición, uso de servicios, estado civil y funcionamiento/discapacidad según el Estudio Nacional de Salud Mental-Colombia
}

\author{
José A. Posada-Villa, MD, Jenny P. Buitrago-Bonilla, TS, Yanithza Medina- \\ Barreto, TS, Marcela Rodríguez-Ospina. MD
}

Universidad Colegio Mayor de Cundinamarca

Correspondencia: hmontoyap@yahoo.com

Recibido: 24-08-06 / Aceptado: 27-09-06

\begin{abstract}
Resumen
Los resultados presentados en este artículo hacen parte de la encuesta mundial de salud mental de la Oorganización Mundial de la Salud, en la que se explora entre otros aspectos, la distribución por edad, género, variaciones por regiones, edad de inicio y uso de servicios asociados a los trastornos de ansiedad en Colombia. El diseño muestral fue de tipo probabilístico, multietápico, estratificado, en población urbana no institucionalizada de 60 municipios representativos del país, en personas entre 18-65 años de edad.

En Colombia los trastornos de ansiedad son de mayor prevalencia que otros trastornos mentales. El 19.3\% de la población entre 18-65 años de edad reporta haber tenido alguna vez en la vida un trastorno de ansiedad. Estos son más frecuentes entre las mujeres (21.8\%). Dentro de los trastornos de ansiedad, la fobia específica es la de mayor prevalencia de vida con $13.9 \%$ en mujeres y $10.9 \%$ en hombres, seguido por la fobia social que es similar en ambos géneros con un $5 \%$ de prevalencia de vida. Por otra parte, la edad de inicio de estos trastornos fluctúa entre los 7 años en el trastorno de fobia específica y los 28 años para el trastorno de estrés post traumático.

Comparando las cinco regiones en las que se dividió el territorio nacional para la aplicación de la encuesta, se encontró que la Región Pacífica y Bogotá D.C son las que presentan la prevalencia más elevada de trastorno de ansiedad alguna vez en la vida con $22.0 \%$ y $21.7 \%$ respectivamente. La Región Atlántica presenta la menor prevalencia (14.9\%). Respecto a las medidas sobre funcionamiento/discapacidad, el trastorno de estrés postraumático es el que genera una mayor discapacidad y siguen en su orden el trastorno de angustia, el trastorno de ansiedad generalizada, la Fobia social y la Agorafobia sin pánico.

Con relación a los trastornos de ansiedad solo el 1.9\% de la población que los sufre acude al psiquiatra para tratamiento y en contraste, el $5.6 \%$ de estos acuden al médico general. Es significativo que el 3.9\% prefieren acudir a tratamientos de medicina alternativa.
\end{abstract}

Palabras claves: agorafobia, ansiedad, desordenes fóbicos, fobia, fobia social, salud mental. 


\begin{abstract}
The results presented in this article are part of the world survey of mental health by the WHO. It explores among other aspects the distribution by age and sex, variations by regions, age of start, and use of services related to anxiety disorders in Colombia. The sample design was probabilistic multileveled and stratified applied to an urban population between 18-65 years of age in 60 representative municipalities of the country, using the Composite International Diagnostic Interview (CIDI).

In Colombia the anxiety disorders are of greater prevalence than other mental disorders. The $19.3 \%$ of the population between 18 and 65 years of age reports to have had an anxiety disorder some time in life. These are more frequent among women $(21.8 \%)$. Within the anxiety disorders, the specific phobia is that of greater life prevalence with $13.9 \%$ in women and $10.9 \%$ in men, followed by the social phobia that is similar in both sexes with a $5 \%$ life prevalence. On the other hand the age of start of these disorders fluctuates between 7 years for the specific phobia disorder and 28 years for the post traumatic stress disorder.

Comparing the five regions in which the national territory was divided for the application of the survey, it was found that the Pacific Region and Bogotá D.C. show the highest prevalence of time any anxiety disorder in life with $22.0 \%$ and $21.7 \%$ respectively. The Atlantic Region presents the smallest prevalence (14.9\%). With respect to the measures on operation/disability, the post-traumatic stress disorder is the one that generates a greater disability followed in order by anxiety disorder, generalized anxiety disorder, social phobia, and agoraphobia without panic. With relation to anxiety disorders, only $1.9 \%$ of the population that suffers it goes to the psychiatrist for treatment; in contrast, $5.6 \%$ of these go to the general practitioner. It is significant that $3.9 \%$ prefer to respond to alternative medicine treatment.
\end{abstract}

Key words: agoraphobia, anxiety, mental health, phobic, phobic disorders, social phobic.

\section{Introducción}

Según la información arrojada por el Estudio de carga global de la Enfermedad, 1990, Organización Mundial de la Salud (OMS), que incluyó los indicadores de discapacidad además de los de mortalidad, cinco de las diez primeras causas de discapacidad a nivel mundial son psiquiátricas. Esto cambió la manera de ver las enfermedades mentales y las posicionó como una prioridad en salud pública.

De acuerdo con los cambios demográficos (crecimiento de la población y continuo desplazamiento del campo a la ciudad) ligados a la urbanización acelerada y desordenada que se ha generado y asociada a los fenómenos de violencia, se han modificado los estilos de vida. Estos cambios han generado impacto en la salud tanto a nivel físico como mental.

La OMS, en 2.001 dedicó su reporte anual a la salud mental (2) con el fin de posicionar esta como un problema importante de salud pública e invitó a los gobiernos a participar en la Encuesta mundial de salud mental (EMSM) que tiene por propósito evaluar la situación de salud mental en países con diferentes niveles de desarrollo, determinar las necesidades de servicio de atención y orientar las políticas de salud pública en el tema.

Como parte de esta iniciativa, se realizó el Estudio Nacional de Salud Mental Colombia-2003 (ENSM), financiado por el Ministerio de la Protección Social. Este busca conocer la extensión de la enfermedad mental en el país, los trastornos más frecuentes, la población que los padece, las variaciones regionales, la distribución por edad y género, el uso de servicios y el funcionamiento/discapacidad asociada a ellos.

Dentro de los trastornos mentales abordados por el ENSM y de mayor interés por el incremento en su prevalencia, se encuentran los trastornos de ansiedad (trastorno de angustia, ansiedad generalizada, fobia social, fobia específica, agorafobia y estrés postraumático). 
En concordancia con lo previsto por la OMS (3), la prevalencia de los trastornos de ansiedad está aumentando a nivel mundial en relación estrecha con problemas como el aumento de la violencia, la persistencia de la pobreza y el consumo de sustancias psicoactivas entre otros estresantes propios de la sociedad actual.

Este incremento en la prevalencia se comprobó a nivel nacional. Así, según el Estudio de salud mental y consumo de sustancias psicoactivas Colombia 1993 (4), que aunque no es comparable con el actual por la metodología utilizada, reportó $9.6 \%$ de prevalencia para el trastorno de ansiedad en contraste con el 19.3\% reportado por el ENSM, Colombia 2003. Es decir que para nuestro país, en un periodo de una década, la prevalencia de estos trastornos se duplicó y se posicionó como el trastorno mental con mayor prevalencia en nuestro medio.

Por lo anterior, este articulo se centra en hacer una descripción detallada de los resultados del ENSM en lo referente a los trastornos de ansiedad, describiendo la distribución por sexo, edad de aparición, uso de servicios y discapacidad asociada a ellos, dada su gran importancia y la urgencia de su abordaje desde el sector de la salud pública.

\section{Materiales y métodos}

\section{Muestra}

El Estudio nacional de salud mental Colombia 2003 (ENSM), utilizó una muestra probabilística con una población civil, no institucionalizada, de 18 a 65 años, en zonas urbanas. Las cinco regiones geográficas de Colombia fueron: Bogotá D.C, Región Atlántica (Córdoba, Sucre, Bolívar, Magdalena, César, Guajira); Región Pacifica (Chocó, Valle del Cauca, Cauca, Nariño); Región Central (Caquetá, Huila, Tolima, Quindío, Risaralda, Caldas y Antioquia) y Región Oriental (Meta, Cundinamarca, Boyacá, Santander, Norte de Santander, Arauca), (5).
Este diseño muestral se basó en la Muestra Maestra del Ministerio de la Protección Social (6) y tiene un diseño probabilístico, por conglomerados, multietápico y estratificado.

La muestra sobre la que se obtuvieron los resultados motivo de esta publicación, estuvo conformada por 60 municipios de los 25 departamentos del país, 420 manzanas, 5.526 hogares urbanos de los diferentes estratos socioeconómicos y 4.544 adultos entre 18 y 65 años.

El trabajo de campo se realizó en el periodo comprendido entre Agosto y Diciembre del 2003, con un equipo conformado por 39 entrevistadores, 11 supervisores locales y 2 supervisores nacionales, todos debidamente entrenados en la aplicación del instrumento y manejo de la información.

\section{Instrumento}

El instrumento utilizado para el estudio EMSM 2000, es el Composite International Diagnostic Interview CIDI-OMS (7), de la cual existen dos versiones: la de aplicación: en papel y lápiz (PAPI) y la versión computarizada (CAPI) por sus siglas en inglés; estas dos versiones son iguales en su contenido y permiten la comparación de datos entre los diferentes países participantes en el estudio; en Colombia se empleo la versión computarizada CAPI, para lo que fue necesario contar con el software Blaise (8) instalado en computadoras portátiles como herramienta de trabajo del equipo encuestador, aplicándola bajo la modalidad de cara a cara.

La encuesta estaba dividida en parte I y parte II. La selección entre estas dos estuvo definido por los resultados del primer módulo aplicado (tamizaje) que contiene preguntas que indican la posibilidad de que alguno de los trastornos indagados se encuentren presentes en el individuo, el cual determinó el rumbo de la entrevista para aplicar la parte II con exploración a profundidad de la patología estudiada. Este diseño tuvo por propósito potenciar la obtención de información y minimizar el tiempo de entrevista. 
Tabla 1.Características demográficas de los participantes del estudio. Estudio Nacional de Salud Mental. Colombia 2003.

\begin{tabular}{ccc}
\hline $\begin{array}{c}\text { Características } \\
\text { demográficas }\end{array}$ & Porcentaje & se \\
\hline $\begin{array}{c}\text { Sexo } \\
\text { Masculino }\end{array}$ & 43.8 & 1.5 \\
Femenino & 56.1 & 1.5 \\
Edad & & \\
$18-29$ & 37.0 & 1.6 \\
$30-44$ & 35.3 & 1.5 \\
$45-59$ & 22.2 & 1.2 \\
$60-65$ & 5.3 & 0.5 \\
\hline
\end{tabular}

Fuente: Informe descriptivo ENSM. Colombia 2003.Ministerio de la Protección Social.

La CIDI-OMS proporciona diagnósticos de alguna vez en la vida, durante los últimos doce meses y durante los últimos treinta días según criterios del DSM-IV y CIE-10 para 17 diagnósticos principales y 6 secundarios. Dentro de los trastornos de ansiedad incluidos en el instrumento se analizaron los Trastornos de angustia, Agorafobia sin trastorno de angustia, Fobia social, Fobia especifica, Trastorno de ansiedad generalizada y Trastorno de estrés postraumático.

Por tratarse de un instrumento estándar para todos los países, se realizo la traducción del instrumento al español, siguiendo las guías desarrolladas por la OMS para tal fin (9).

\section{Análisis}

Los datos de la encuesta, se obtuvieron a través de una muestra multietápica y estratificada; posteriormente se realizó una post estratificación para la población colombiana teniendo en cuenta los cambios poblacionales después del censo de 1993, basados en las últimas encuestas de hogares realizadas por el Departamento Administrativo Nacional de Estadísticas DANE (10).

El análisis se realizó a partir de la base de datos aprobada por OMS y la Universidad de Harvard. Para calcular las prevalencias se empleo una tabulación cruzada sencilla. El método de Kaplan - Meier (11) se utilizó para generar las curvas de edad de inicio de los trastornos. Los errores estándar de estadística descriptiva (alguna vez en la vida, últimos 12 meses y últimos 30 días) se estimaron usando el método de series de Taylor (12), implementado en el sistema de software SUDAAN (13). Los coeficientes de regresión logística se transformaron en ORs (razón de disparidad) reportados en la parte inferior con intervalos de confianza ajustados al 95\%. Los test multivariados están basados en las pruebas de Wald (14). La significancia estadística se basó en una prueba de un nivel de significancia del 0.05 .

\section{Resultados}

\section{Características de la población estudiada.}

La Tabla 1 presenta la distribución por sexo y edad de la muestra. Cerca del 56\% fue del sexo femenino y aproximadamente el $37 \%$ se ubicó en el grupo de edad más joven (18 - 29 años).

\section{Descripción general de la prevalencia de los} trastornos mentales

En la Tabla 2 se presentan las prevalencias de vida distribuidas por género de los 3 grupos de trastornos mentales según el DSM -IV indagados en el estudio, evidenciando que los Trastornos de ansiedad son los que presentan la mayor prevalencia con un $19.3 \%$.

En las Tablas 3 y 4 se presentan las prevalencias de los Trastornos de ansiedad, para doce meses y últimos 30 días, que son de $11.7 \%$ y $5.1 \%$ respectivamente. 
Tabla 2. Prevalencia de vida de trastornos según DSM-IV Colombia 2003.

\begin{tabular}{lcccccc}
\hline \multicolumn{1}{c}{ Diagnostico } & \multicolumn{2}{c}{ Hombres } & \multicolumn{2}{c}{ Mujeres } & \multicolumn{2}{c}{ Total } \\
& $\%$ & $\begin{array}{c}\% \\
(\mathrm{se})\end{array}$ & $\%$ & $\begin{array}{c}\% \\
(\mathrm{se})\end{array}$ & $\%$ & $\begin{array}{c}\% \\
\text { (se) }\end{array}$ \\
\hline Trastornos del estado de & 11.7 & 1.1 & 17.5 & 1.1 & 15.0 & 0.9 \\
ánimo. & & & & & & \\
Trastornos de ansiedad & 16.0 & 1.3 & 21.8 & 1.5 & 19.3 & 1.1 \\
Trastornos relacionados & 10.6 & 1.7 & 2.6 & 0.4 & 10.6 & 0.8 \\
con sustancias & & & & & & 1.6 \\
Cualquier trastorno & 41.0 & 2.1 & 39.5 & 2.0 & 40.1 & 1.6 \\
\hline \hline
\end{tabular}

Fuente: Informe descriptivo ENSM. Colombia 2003.Ministerio de la Protección Social.

Tabla 3. Prevalencia de 12 meses de trastornos según DSM-IV. Colombia 2003.

\begin{tabular}{lcccccc}
\hline \multicolumn{1}{c}{ Diagnostico } & \multicolumn{2}{c}{ Hombres } & \multicolumn{2}{c}{ Mujeres } & \multicolumn{2}{c}{ Total } \\
& $\%$ & $\begin{array}{c}\% \\
\text { (se) }\end{array}$ & $\%$ & $\begin{array}{c}\% \\
\text { (se) }\end{array}$ & $\%$ & $\begin{array}{c}\% \\
\text { (se) }\end{array}$ \\
\hline $\begin{array}{l}\text { Trastornos del estado de } \\
\text { ánimo }\end{array}$ & 4.5 & 0.7 & 8.7 & 0.8 & 6.9 & 0.6 \\
$\begin{array}{l}\text { Trastornos de ansiedad } \\
\text { Trastornos relacionados }\end{array}$ & 9.1 & 1.1 & 13.7 & 1.3 & 11.7 & 0.9 \\
con sustancias & 5.8 & 1.0 & 0.8 & 0.2 & 3.0 & 0.4 \\
Cualquier trastorno & 15.6 & 1.4 & 16.3 & 1.5 & 16.0 & 1.2 \\
\hline
\end{tabular}

Fuente: Informe descriptivo ENSM. Colombia 2003. Ministerio de la Protección Social.

Tabla 4. Prevalencia de últimos 30 días de trastornos según DSM-IV. Colombia 2003.

\begin{tabular}{lcccccc}
\hline \multicolumn{1}{c}{ Diagnostico } & \multicolumn{2}{c}{ Hombres } & \multicolumn{2}{c}{ Mujeres } & \multicolumn{2}{c}{ Total } \\
& $\%$ & $\begin{array}{c}\% \\
\text { (se) }\end{array}$ & $\%$ & $\begin{array}{c}\% \\
\text { (se) }\end{array}$ & $\%$ & $\begin{array}{c}\% \\
\text { (se) }\end{array}$ \\
\hline Trastornos del estado de & 0.8 & 0.2 & 3.1 & 0.4 & 2.1 & 0.3 \\
Ánimo & & & & & & \\
Trastornos de ansiedad & 3.7 & 0.7 & 6.2 & 0.9 & 5.1 & 0.6 \\
Trastornos relacionados & 1.8 & 0.4 & 0.2 & 0.1 & 0.9 & 0.2 \\
con sustancias & & & & & & 0.7 \\
Cualquier trastorno & 5.8 & 0.8 & 8.7 & 1.0 & 7.4 & 0.7 \\
\hline
\end{tabular}

Fuente: Informe descriptivo ENSM. Colombia 2003. Ministerio de la Protección Social.

Prevalencia para 12 meses y últimos 30 días en los trastornos de ansiedad específicos

En la Tabla 5 se muestran las prevalencias de vida, de doce meses y de últimos 30 días para los trastornos específicos de ansiedad con la distribución por género. Este permite ver que la Fobia específica es el trastorno que presenta mayor prevalencia de vida, 12 meses y 30 días siendo mayor en mujeres.

Las edades de inicio de los trastornos específicos de ansiedad son las siguientes: la Fobia específica con mediana de 7 años, es el trastorno de ansiedad de aparición más temprana, seguido por la Fobia social con mediana de 14 años, Agorafobia 16 años, Trastorno de angustia 18 años, Trastorno de ansiedad generalizada 20 años y Trastorno de estrés postraumático 28 años.

En la Figura 1 se presenta la prevalencia de alguna vez en la vida de los Trastornos de ansiedad comparada en las cinco regiones en las que se dividió el territorio nacional para efectos de la aplicación y análisis del estudio. 
Tabla 5. Prevalencia de vida, 12 meses y 30 días de los trastornos de ansiedad específicos por sexo.

\begin{tabular}{|c|c|c|c|c|c|c|c|c|c|}
\hline \multirow[b]{2}{*}{ Diagnóstico } & \multicolumn{3}{|c|}{$\begin{array}{c}\text { Prevalencia de vida } \\
\%\end{array}$} & \multicolumn{3}{|c|}{ Prevalencia de 12 meses \% } & \multicolumn{3}{|c|}{$\begin{array}{c}\text { Prevalencia de } 30 \text { días } \\
\%\end{array}$} \\
\hline & Hombres & Mujeres & Total & Hombres & Mujeres & Total & Hombres & Mujeres & Total \\
\hline $\begin{array}{l}\text { Trastorno de } \\
\text { angustia }\end{array}$ & 0.6 & 1.7 & 1.2 & 0.3 & 0.9 & 0.6 & 0.1 & 0.5 & 0.3 \\
\hline $\begin{array}{l}\text { Agorafobia sin } \\
\text { angustia }\end{array}$ & 1.5 & 3.3 & 2.6 & 1.3 & 1.7 & 1.5 & 0.4 & 0.4 & 0.4 \\
\hline Fobia Social & 5.1 & 5.1 & 5.1 & 2.1 & 2.8 & 2.5 & 0.8 & 0.7 & 0.7 \\
\hline Fobia específica & 10.9 & 13.9 & 12.6 & 4.9 & 6.9 & 6.0 & 2.2 & 2.7 & 2.5 \\
\hline $\begin{array}{l}\text { Trastorno de } \\
\text { Ansiedad } \\
\text { generalizada }\end{array}$ & 1.5 & 1.2 & 1.3 & 0.9 & 0.5 & 0.7 & 0.5 & 0.2 & 0.3 \\
\hline $\begin{array}{l}\text { Trast. Estrés } \\
\text { postraumático }\end{array}$ & 0.8 & 2.5 & 1.8 & 0.1 & 0.7 & 0.5 & 0.1 & 0.3 & 0.2 \\
\hline $\begin{array}{l}\text { Cualquier } \\
\text { trastorno de } \\
\text { ansiedad }\end{array}$ & 16.0 & 21.8 & 19.3 & 9.1 & 13.7 & 11.7 & 3.7 & 6.2 & 5.1 \\
\hline
\end{tabular}

Fuente: Informe descriptivo ENSM. Colombia 2003. Ministerio de la Protección Social.

Tabla 6. Prevalencia de vida de Trastornos de ansiedad según estado civil. Colombia 2003

\begin{tabular}{|c|c|c|c|c|c|c|c|c|}
\hline \multirow[t]{2}{*}{ Diagnóstico } & \multicolumn{2}{|c|}{ Casado- Unión } & \multicolumn{2}{|c|}{ Separado- } & \multicolumn{2}{|c|}{ Soltero } & \multicolumn{2}{|c|}{ Total } \\
\hline & $\begin{array}{l}\text { Libre } \\
\%\end{array}$ & $\begin{array}{l}\% \\
\text { (se) }\end{array}$ & $\begin{array}{l}\text { Viud } \\
\%\end{array}$ & $\%$ (se) & $\%$ & $\%$ (se) & $\%$ & $\%$ (se) \\
\hline Tras. de angustia. & 1.2 & 0.3 & 1.4 & 0.6 & 1.2 & 0.4 & 1.3 & 0.2 \\
\hline $\begin{array}{l}\text { Agorafobia sin crisis de } \\
\text { angustia. }\end{array}$ & 2.6 & 0.5 & 3.2 & 1.1 & 2.1 & 0.5 & 2.5 & 0.4 \\
\hline Fobia social & 4.5 & 0.5 & 5.3 & 1.3 & 6.1 & 1.0 & 5.1 & 0.5 \\
\hline $\begin{array}{l}\text { Fobia especifica } \\
\text { Trastorno de Ansiedad } \\
\text { generalizada }\end{array}$ & $\begin{array}{l}12.6 \\
1.2\end{array}$ & $\begin{array}{l}0.9 \\
0.4\end{array}$ & $\begin{array}{l}12.6 \\
2.3\end{array}$ & $\begin{array}{l}2.5 \\
0.8\end{array}$ & $\begin{array}{l}12.4 \\
1.1\end{array}$ & $\begin{array}{l}1.7 \\
0.3\end{array}$ & $\begin{array}{l}12.5 \\
1.3\end{array}$ & $\begin{array}{l}0.8 \\
0.3\end{array}$ \\
\hline $\begin{array}{l}\text { Trastorno de estrés } \\
\text { postraumático }\end{array}$ & 1.5 & 0.4 & 2.4 & 0.8 & 2.0 & 1.1 & 1.7 & 0.4 \\
\hline $\begin{array}{l}\text { Cualquier trastorno de } \\
\text { Ansiedad }\end{array}$ & 18.4 & 1.2 & 21.0 & 3.1 & 19.7 & 2.1 & 19.2 & 1.0 \\
\hline
\end{tabular}

Fuente: Informe descriptivo ENSM. Colombia 2003.Ministerio de la Protección Social.

Tabla 7. Distribución por tipo de vinculación al sistema de seguridad social en salud por trastorno especifico de ansiedad. Colombia 2003.

\begin{tabular}{|c|c|c|c|c|c|c|c|c|}
\hline \multirow[b]{2}{*}{ Diagnóstico } & \multicolumn{2}{|c|}{ Contributivo } & \multicolumn{2}{|c|}{ Sin régimen } & \multicolumn{2}{|c|}{ Subsidiado } & \multicolumn{2}{|c|}{ Total } \\
\hline & $\%$ & $\begin{array}{c}\% \\
\text { (se) }\end{array}$ & $\%$ & $\begin{array}{c}\% \\
\text { (se) }\end{array}$ & $\%$ & $\begin{array}{c}\% \\
\text { (se) }\end{array}$ & $\%$ & $\begin{array}{c}\% \\
\text { (se) }\end{array}$ \\
\hline Trastorno de angustia & 1.4 & 0.5 & 1.0 & 0.3 & 1.5 & 0.4 & 1.3 & 0.2 \\
\hline $\begin{array}{l}\text { Agorafobia sin crisis de } \\
\text { Angustia. }\end{array}$ & 3.3 & 0.9 & 2.0 & 0.3 & 2.4 & 0.6 & 2.5 & 0.4 \\
\hline Fobia social & 6.1 & 0.9 & 4.2 & 0.7 & 6.3 & 0.9 & 5.1 & 0.5 \\
\hline Fobia especifica & 13.0 & 1.2 & 11.3 & 1.1 & 13.7 & 1.8 & 12.5 & 0.8 \\
\hline $\begin{array}{l}\text { Trastorno de Ansiedad } \\
\text { generalizada }\end{array}$ & 1.1 & 0.4 & 1.1 & 0.3 & 1.6 & 0.7 & 1.3 & 0.3 \\
\hline Trastorno de estrés postraumático & 1.8 & 0.6 & 0.7 & 0.2 & 3.1 & 1.1 & 1.7 & 0.4 \\
\hline Cualquier Trastorno de Ansiedad & 19.7 & 1.6 & 16.4 & 1.3 & 22.3 & 2.2 & 19.2 & 1.0 \\
\hline
\end{tabular}

Fuente: Informe descriptivo ENSM. Colombia 2003. Ministerio de la Protección Social. 


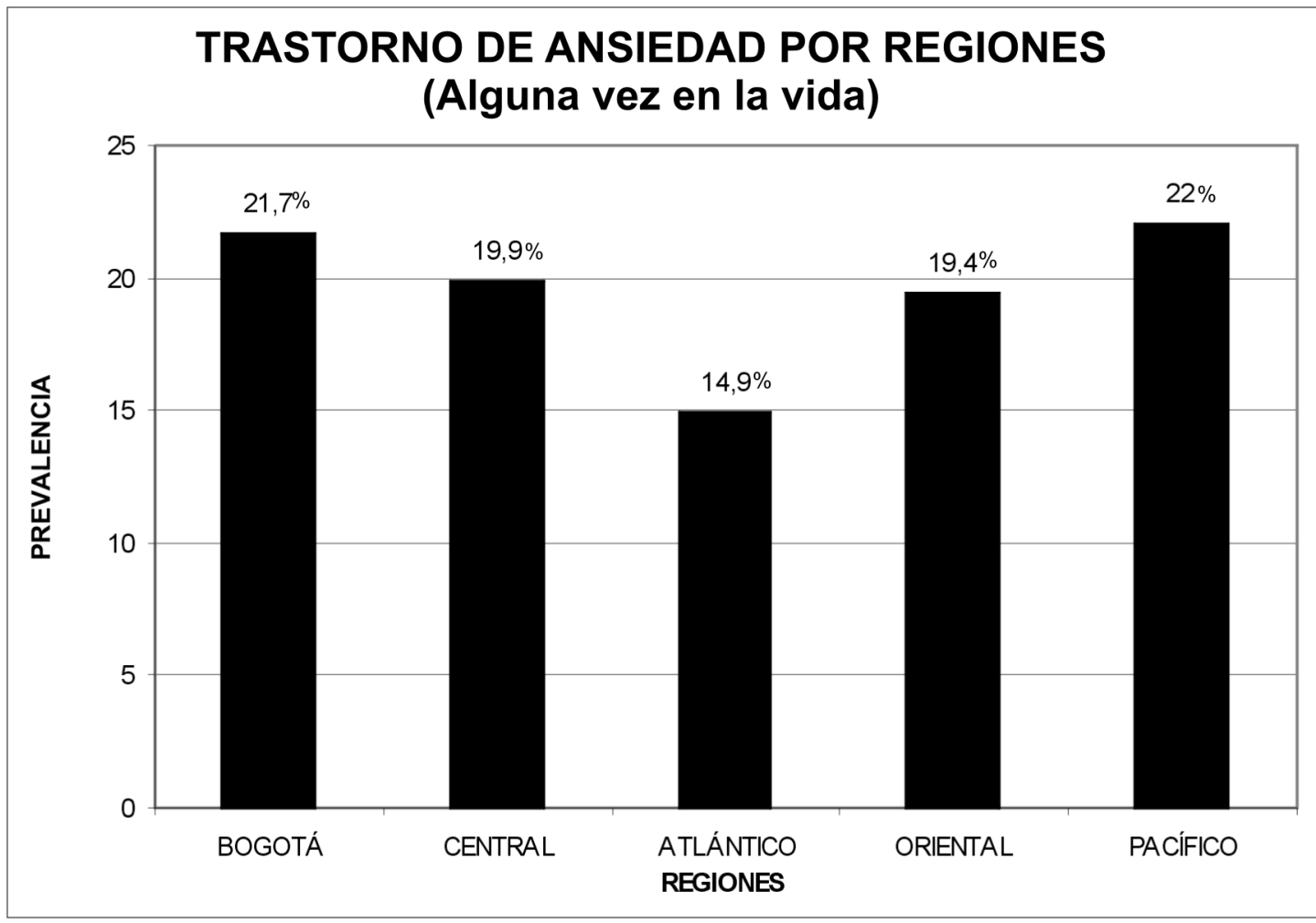

Figura 1. Trastorno de ansiedad por regiones (alguna vez en la vida)

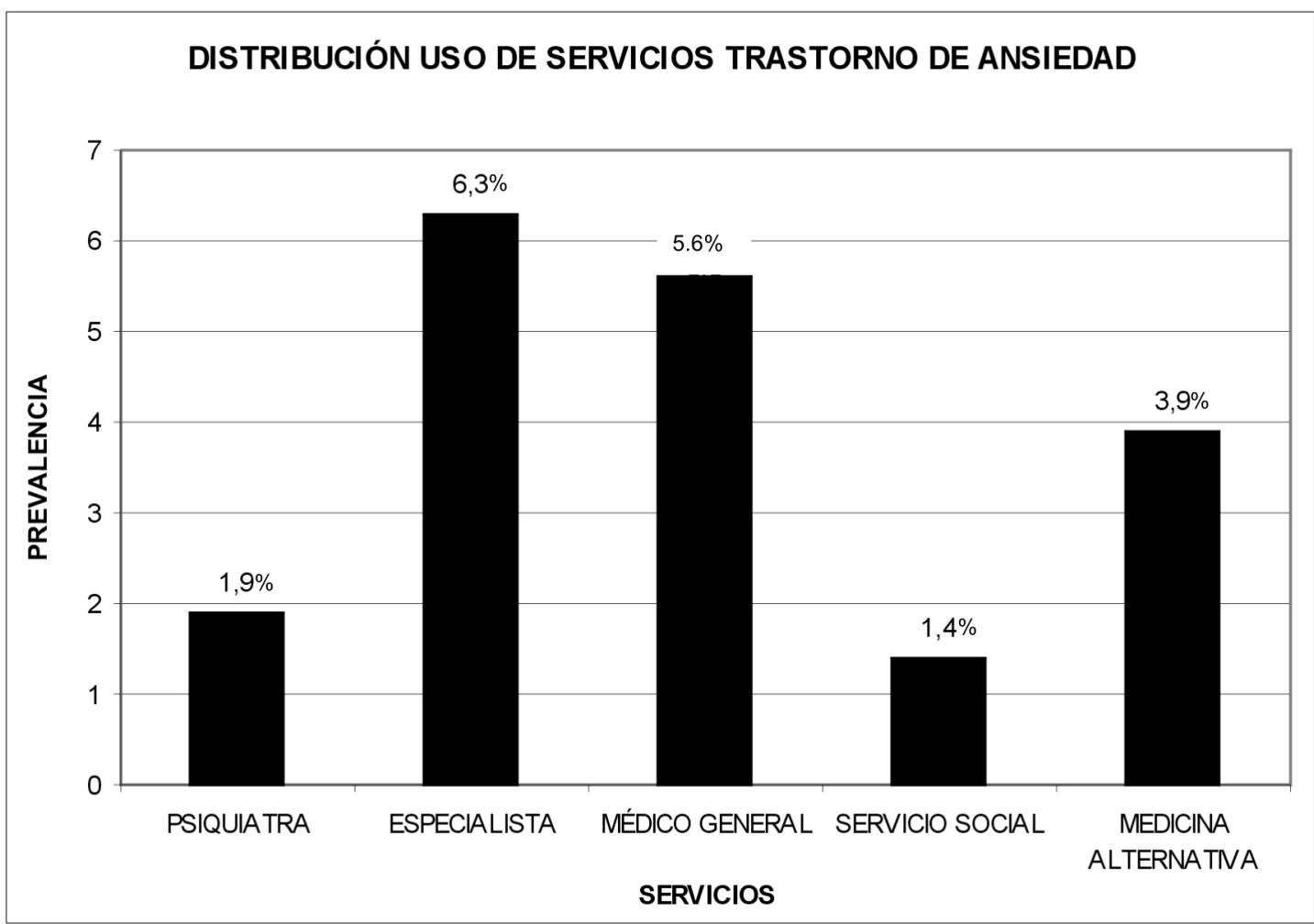

Figura 2. Distribución uso de servicios trastorno de ansiedad 
En primer lugar, se observa que la Región Pacifica y Bogotá D.C. son las de mayor prevalencia de vida con $22 \%$ respectivamente, encontrándose la menor prevalencia en la Región Atlántica (15\%).

En la Tabla 6 se presenta la prevalencia de alguna vez en la vida de los trastornos de ansiedad de acuerdo al estado civil, con distribuciones muy homogéneas entre los diferentes trastornos.

La fobia social es la única que se aparta de esta característica, ya que presenta la mayor prevalencia en el grupo de solteros con $6.1 \%$, con respecto a la prevalencia en el grupo de casados correspondiente a $4.5 \%$.

En relación con la medida de funcionamiento/ discapacidad de las personas que padecen alguno de estos trastornos. Se observa que el menor grado de discapacidad está dado por la agorafobia sin pánico y la mayor discapacidad se observa con el trastorno de estrés post traumático.

En la Tabla 7 se presenta la prevalencia de vida de los trastornos de ansiedad distribuidos por tipo de vinculación al Sistema de Seguridad Social, encontrándose la mayor prevalencia de cualquier trastorno de ansiedad en el grupo de población perteneciente al régimen subsidiado con $28.6 \%$ frente a $26.7 \%$ del régimen contributivo y $20.7 \%$ para la población no vinculada, quienes de manera general presentan las menores prevalencias para los trastornos de ansiedad.

En los trastornos específicos llama la atención que en general la distribución en los tres grupos es homogénea, excepto para el trastorno de estrés postraumático en el que la población perteneciente al régimen subsidiado presenta una prevalencia de 3.1\% frente a $1.8 \%$ del contributivo y $0.7 \%$ de la población vinculada.

En la Figura 2 se muestra la distribución de uso de servicios por trastornos de ansiedad, teniendo en cuenta que "otro especialista" hace referencia a psicólogos, psicoterapeutas, enfermeras, trabajadores sociales y orientadores en escenarios de salud mental. En esta se puede observar que el servicio más utilizado por las personas que padecen trastornos de ansiedad es el correspondiente al grupo de "otros especialistas" con $6.3 \%$, seguido por el de médicos generales. Sin embargo el psiquiatra solo es consultado por el $1.9 \%$ de las personas que consultan por trastornos de ansiedad.

\section{Discusión}

Los resultados presentados en este artículo son de importancia ya que en los informes preliminares se observa que el trastorno de ansiedad es el de mayor prevalencia en Colombia con $19.3 \%$, frente a otros trastornos (estado de ánimo y uso de sustancias). Tres de cada diez mujeres y dos de cada diez hombres han presentado trastorno de ansiedad alguna vez en la vida. La Región Pacífica y Bogotá D.C. muestran la prevalencia más elevada de trastornos de ansiedad alguna vez en la vida. En general los trastornos de ansiedad son los que aparecen a edades más tempranas, seguidos por los trastornos por uso de sustancias y por último los trastornos afectivos.

Uno de los datos más importantes que arroja el estudio es que los trastornos de ansiedad se están presentando en edades tempranas. En cuanto al trastorno de ansiedad, la población consulta más a otros especialistas distintos al psiquiatra, haciendo referencia a psicólogos, psicoterapeutas, enfermeras, trabajadores sociales y orientadores en escenarios de salud mental, seguido por médicos generales.

El trastorno que genera mayor compromiso del funcionamiento/discapacidad es el trastorno de estrés postraumático, y el que genera menor grado es la Agorafobia sin pánico.

Los datos indican que de las personas que sufren algún trastorno de ansiedad, el $20.7 \%$ no accede al Sistema de Seguridad Social en Salud, lo cual hace necesario desarrollar programas para ampliar la cobertura en servicios de salud.

\section{Agradecimientos}

The Colombian National Study of Mental Health (NSMH) is supported by the Ministry of Social Protection, with supplemental support from the 
Saldarriaga Concha Foundation. The ENSM is carried out in conjunction with the World Health Organization World Mental Health (WMH) Survey Initiative. We thank the WMH staff for assistance with instrumentation, fieldwork, and data analysis. These activities were supported by the United States National Institute of Mental Health (R01MH070884), the John D. and Catherine T. MacArthur Foundation, the Pfizer Foundation, the US Public Health Service (R13MH066849, R01-MH069864, and R01 DA016558), the Fogarty International Center (FIRCA R01-TW006481), the Pan American Health Organization, Eli Lilly and Company, Ortho-McNeil Pharmaceutical, Inc., GlaxoSmithKline, and Bristol-Myers Squibb. A complete list of WMH publications can be found at http:// www.hcp.med.harvard.edu/wmh/.

\section{Referencias}

1. World Health Organization (WHO): Composite International Diagnostic Interview (CIDI). Version 15. Geneva; 2000.

2. World Health Organization (WHO). The world health report 2001, mental health: New Understanding. New Hope: Geneva; 2.001.

3. Departamento de Salud y Servicios Humanos EEUU: Salud Mental: cultura, raza y etnias: informe de la subdirección general. Rockville, MD 2001.

4. Posada J., et al. En Estudio Nacional de Salud Mental y Consumo de Sustancias Psicoactivas Ministerio de Salud Colombia 1993.

5. Posada, J., Aguilar, S. et, al. Prevalencia de trastornos mentales y uso de servicios: Resultados preliminares del Estudio nacional de salud mental. Colombia 2003: Revista colombiana de Psiquiatría 2.004 Volumen XXXIII N 3.

6. Ministerio de la Protección Social, Dirección de Salud Pública .Muestra para Estudios en Salud. 2003.

7. World Health Organization (WHO): Composite international diagnostic interview (CIDI). Version 15. Geneva; 2.000.

8. Blaise. Statistic Netherlands, Boorbug/Heerlen. 2003.

9. WHO. Procedures for the development of new language versions of the WHO Composite International Diagnostic Interview (WHO-CIDI). Geneva; 1.998.

10. DANE. Censo 1993. República de Colombia: XVI Censo Nacional de Población y de Vivienda; junio 1996.

11. Kaplan and Meier. Nonparametric estimation from incomplete observations. 1958.

12. Wolter, KM. Introduction to variance estimation, 1985

13. SUDAAN (software). Version 8.0.1. 2002.

14. Murray C. and Lopez A. The global burden of disease, OMS, 1996. 